\title{
Fármacos multidiana en el posible tratamiento de la esclerosis lateral amiotrófica.
}

\author{
Esperanza Bertila Lezana Juberías 1, 2, a, Loreto Martínez-González², Ana Martínez² \\ 1. Unidad de Bioquímica y Biología Molecular, Departamento de Biología de Sistemas, Facultad de Medicina y Ciencias \\ de la Salud, Universidad de Alcalá, 28871. Alcalá de Henares, Madrid, España. 2. Grupo de Química Médica y \\ Biológica traslacional, Departamento de Biología Físico-Química, Centro de Investigaciones Biológicas, CSIC, 28040 \\ Madrid, Madrid, España.
}

a. esperanzalezana@gmail.com

Palabras clave: esclerosis lateral amiotrófica; TDP-43; GSK-3, CK1, TTBK, fármacos multidiana

\section{Resumen}

La esclerosis lateral amiotrófica (ELA) es una enfermedad neurodegenerativa en la que se produce la muerte de las motoneuronas superiores e inferiores. Aunque tiene un origen desconocido y multifactorial, en el $97 \%$ de los pacientes se ha encontrado depósitos de agregados de la proteína TDP-43 (proteína TAR de unión a ADN/ARN). Las funciones de esta proteína nuclear se centran en la regulación del ARN. La proteinopatía de TDP-43 se desencadena con la translocación del núcleo al citoplasma y su hiperfosforilación, lo que da lugar a la acumulación de agregados proteicos y consiguiente degeneración neuronal. Las quinasas que participan en este proceso de fosforilación son: GSK3, CK1, TTBK, CDC7. En este trabajo se propone la modulación de varias quinasas simultáneamente para conseguir un efecto farmacológico más eficaz. Para ello se han evaluado fármacos multidiana, con inhibición dual simultánea en dos de las cuatro quinasas mencionadas, así como combinaciones de monoterapias. Los resultados obtenidos han mostrado sinergia en los tratamientos y validan la utilización de fármacos multidiana en patologías complejas como la ELA.

Cita: Lezana Juberías, Esperanza Bertila; Martínez-González, Loreto; Martínez, Ana (2019) Fármacos multidiana en el posible tratamiento de la esclerosis lateral amiotrófica. dianas 8 (2): e201909fa03. ISSN 18868746 (electronic) journal.dianas.e201909fa03 http://www3.uah.es/dianas?e201909fa03. URI http://hdl.handle.net/10017/15181

Copyright: ( ) Lezana-Juberías EB, Martínez-González L, Martínez A. Algunos derechos reservados. Este es un artículo open-access distribuido bajo los términos de una licencia de Creative Commons ReconocimientoNoComercial-SinObraDerivada 4.0 Internacional. http://creativecommons.org/licenses/by-nc-nd/4.0/

\section{Introducción}

Las enfermedades neurodegenerativas constituyen un grupo de patologías complejo de origen desconocido. La enfermedad de Alzheimer o de Parkinson, la esclerosis lateral amiotrófica y la demencia temporal (FTLD), son algunos de los ejemplos de estas patologías. Factores múltiples como la inflamación, el estrés oxidativo, o la excitotoxicidad, contribuyen a la desintegración paulatina del sistema nervioso central (SNC). El mayor factor de riesgo para todas estas enfermedades es la edad. Por ello y dado el aumento actual de la esperanza de vida, la incidencia de estas patologías en la población está aumentando de manera exponencial, convirtiéndolas en un problema de salud a nivel mundial. Actualmente, únicamente existen tratamientos sintomáticos, por lo que el descubrimiento de fármacos efectivos supone un verdadero reto terapéutico[1].

\section{Esclerosis lateral amiotrófica}

La esclerosis lateral amiotrofia (ELA) es una enfermedad de origen desconocido caracterizada por la degeneración progresiva de las neuronas motoras superiores (neuronas de la corteza motora) como de las inferiores (neuronas del tronco cerebral y la medula espinal) [2]. Se conoce también como enfermedad de Charcot, en honor al hombre que describió por primera vez la enfermedad, y presenta entre sus síntomas más comunes: debilidad muscular, espasmos, calambres, disnea y disfagia, que terminan desencadenado en insuficiencia respiratoria y muerte [3].

En Europa, los casos de ELA se encuentran entre 2-3 por cada 100.000 personas siendo la edad de inicio entorno a los 65 años. La enfermedad puede clasificarse en: ELA esporádico (90-95\%) y ELA familiar (5$10 \%$ ). La patología esporádica está asociada con la presencia de múltiples desencadenantes como son: excitotoxicidad por glutamato, estrés oxidativo, alteraciones en el transporte axonal, así como daño mitocondrial. El ELA familiar, se caracteriza por la presencia de antecedentes familiares, siendo el 70\% de los casos mutaciones en los genes C9orf72, TARDBP (proteína TAR de unión a ADN/ARN), SOD1 (superóxido dismutasa) y FUS (proteína FUS). En ambos casos de ELA, se ha encontrado que el 97\% de los pacientes presentan agregados de la proteína TDP-43[2,3]. Es por esto, por lo que el presente estudio se centra en la proteinopatía de TDP-43. 


\section{Proteinopatía de TDP-43}

TDP-43 es una proteína muy conservada de 414 aminoácidos, codificada por TARDBP. Se expresa de forma ubicua por todo el organismo. En cuanto a su estructura, está constituida por dos motivos de reconocimiento de ARN (RRM1) y (RRM2), una secuencia de localización nuclear (NLS), una señal de exportación nuclear (NES) y un dominio rico en glicina en el carboxilo terminal el cual media las interacciones proteína-proteína. Es en esta última región, donde se han identificado la mayoría de mutaciones esporádicas y familiares asociadas a ALS, por lo que alteraciones en su funcionalidad repercutirían en el desarrollo de la neurodegeneración [4].

Perteneciente a la familia de las ribonucleoproteínas heterogénea nucleares (hnRNP), entre sus funciones destacan las siguientes: transcripción, traducción, transporte de ARNm, estabilización del ARNm, microARN (miARN) y procesamiento largo de ARN no codificante. Aunque es una proteína predominantemente nuclear también puede localizarse en el citoplasma, donde interacciona con proteínas citoplasmáticas encargadas de la traducción del ARNm [5].

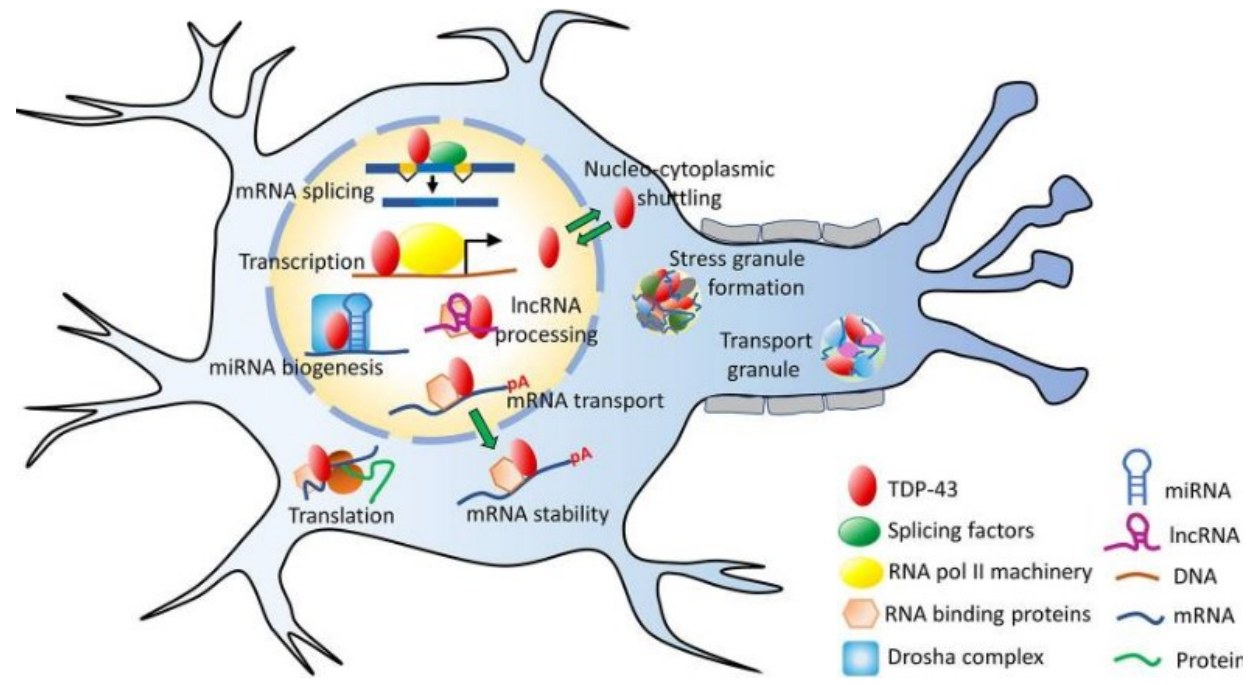

Figura 1.- Funciones del TDP-43. TDP-43 realiza varios procesos relacionados con el ARNm en el núcleo, como la transcripción, el empalme, el mantenimiento de la estabilidad del ARN, así como el procesamiento de miARN. Aunque es predominantemente una proteína nuclear, puede translocarse al citoplasma. Es en el citoplasma, donde TDP-43 participa en la formación de gránulos de estrés, en la formación de gránulos de transporte de ribonucleoproteína (RNP), la traducción y otros procesos [5].

TDP-43 sufre una gran variedad de modificaciones postraduccionales, tales como fosforilación, ubiquitinación, acetilación, y oxidación de la cisteína. La patología de TDP-43 hace referencia a la deposición de fragmentos de TDP-43 ubiquinizados e hiperfosforilados en forma de agregados en el citoplasma. Produciéndose así, la ausencia y la funcionalidad de la proteína en el núcleo. TDP-43 tiene múltiples residuos donde puede ser fosforilados (41 residuos de serina, 15 de treonina y 8 de tirosina). Siendo los sitios específicos de fosforilación serina 409 y serina 410 . Se han descrito una serie de quinasas implicadas en la fosforilación de TDP-43 entre las que se encuentran, GSK3, CK1, TTBK1, CDC7 [5].

\section{GSK-3}

La glucógeno sintasa quinasa 3 (GSK3) es una serina treonina quinasa, constituida por dos isoformas, GSK-3 $\alpha$ y GSK-3 $\beta$, está implicada en el desarrollo neuronal, transcripción, división, supervivencia y muerte celular, así como en las vías de Wnt, Hedgehog y glucógeno. Aunque comparten una alta homología en el dominio quinasa, solamente muestran un $36 \%$ de coincidencia en la zona carboxilo terminal. GSK-3 $\beta$ se localiza de forma abundante en el sistema nervioso central, donde fosforila a proteínas asociadas a microtúbulos, actividades aberrantes de la proteína desencadenan enfermedades neurodegenerativas tales como Alzheimer y ELA. La hiperactivación de GSK-3 $\beta$ es por tanto un mecanismo desencadenante de la enfermedad [6].

\section{CK1}

La proteína quinasa 1 (CK1) es capaz de fosforilar in vitro a la proteína TDP-43 [7]. Estudios realizados en linfoblastos de pacientes con ELA y FTLD, han demostrado, que los inhibidores de CK1 modulan la homeostasis de TDP-43 [8].

CK1, es una proteína que se expresa de forma ubicua en organismos eucariotas, está constituida por siete isoformas, siendo CK1 $\delta$ la que produce mayor número de fosforilaciones en sitios diferentes. Participa en 
transporte de membrana, regulación de la reparación de $\mathrm{ADN}$, morfología celular, modulación de la vía Wnt/B-catenina y vía Hedgehog, regulación de los ritmos circadianos. A nivel de patologías neurodegenerativas, CK1 participa en la fosforilación de tau, TDP-43, $\alpha$-sinucleína [9].

\section{TTBK1}

La quinasa de la proteína tau y de la tubulina (TTBK) presenta dos isoformas TTBK1 Y TTBK2 y estructuralmente pertenece a la familia de las caseína quinasas. Son proteínas muy conservadas que fosforilan en los residuos de serina, treonina y tirosina. Aunque difieren en el dominio regulador comparten un dominio homólogo en el carbono terminal [10].

TTBK1 se expresa mayoritariamente en el sistema nervioso central, aunque también lo hace de manera minoritaria en los testículos. Por su parte, TTBK2 se localiza de manera más heterogénea, encontrándose en hígado, músculo esquelético, páncreas, corazón y cerebro, siendo el lugar predominante los testículos. A nivel ciliar está implicado en la iniciación de la ciliogénesis así como en el control del tráfico y longitud ciliar. TTBK2 también interviene en la regulación de los transportadores acoplados a sodio. Es de gran relevancia el papel que ambas isoformas juegan en el proceso de fosforilación de los microtúbulos tau, que son característicos en la enfermedad de Alzheimer. Así mismo, se ha visto que son capaces de fosforilar directamente a TDP-43 en experimentos in vitro. Dada la selectividad por el SNC de TTBK1, puede ser una importante diana terapéutica, ya que ejerce una acción dual en tau y en TDP$43[10]$.

\section{Terapéutica de la esclerosis lateral amiotrófica}

Actualmente, solo dos fármacos se encuentran disponibles para el tratamiento paliativo de la ELA en el mercado, Riluzol y Edaravone. El mecanismo de acción del primero de ellos se basa en el bloqueo de los canales controlados por voltaje en las neuronas presinápticas, lo que se traduce en una reducción de transmisión glutamatérgica. En los ensayos realizados, se observa que, aunque aumenta la supervivencia hasta los tres meses no es capaz de ejercer ningún efecto sobre la fuerza del músculo. Edavarone, aprobado únicamente por la FDA, se piensa que actúa como agente antioxidante, es capaz de retrasar la progresión de la enfermedad en pacientes seleccionados de forma restringida. Estos dos fármacos, no son capaces de proporcionar un tratamiento efectivo, por lo que se hace necesario e indispensable el descubrimiento y desarrollo de nuevos fármacos que frenen el avance de la enfermedad [2].

La ELA, como todas las enfermedades neurodegenerativas, es una patología compleja, heterogénea y multifactorial, cuyo tratamiento difícilmente podría conseguirse utilizando una monoterapia. Precisamente, para encontrar una estrategia terapéutica eficaz, surge la idea de utilizar fármacos multidiana para el tratamiento de patologías multifactoriales. Los fármacos multidiana son entidades químicas únicas, que presentan una actividad múltiple modulando de manera simultánea diferentes dianas [11]. Atendiendo a su diseño, los fármacos multidiana se pueden clasificar en compuestos híbridos, cuando dos o más fármacos con distintas actividades se conectan mediante un espaciador estable o metabolizable [12] o en compuestos quiméricos, cuando los nuevos fármacos se diseñan a partir de la fusión de farmacóforos presentes en diferentes compuestos activos [13].

Las quinasas que fosforilan a TDP-43, provocan una hiperfosforilación de la proteína, dando lugar a la perdida de la homeostasia y a la aparición de un proceso patológico. Esto hace, que sean unas dianas terapéuticos importantes puesto que juegan un papel indispensable en el desarrollo de las enfermedades neurodegenerativas. Por lo tanto, la hipótesis de trabajo subyacente es que la inhibición de dos quinasas responsables de la fosforilación de TDP-43 simultáneamente podría producir mayores efectos terapéuticos que los generados de manera individual.

El uso de los compuestos duales, tiene además otras ventajas como son, la mayor adherencia terapéutica, la menor dificultad en la formulación (elaboración de una única forma farmacéutica), una farmacocinética más sencilla, así como la disminución de las posibles interacciones entre los fármacos, puesto que la interacción de fármaco-fármaco no existe. Hay que tener en cuenta, que al tratarse de una única molécula y no de dos, evitamos la polimedicación del paciente, y conseguimos aumentar la potencia del efecto con una menor cantidad de dosis administrada. Todo esto, proporciona una reducción de los efectos adversos junto con una mayor seguridad para el paciente, además de que esta alternativa supondría un menor coste para el sistema sanitario.

En este contexto, el grupo de Química médica y Biología translacional, ha diseñado y sintetizado compuestos duales de las quinasas GSK3, CK1 y TTBK1. El objetivo del presente trabajo es comprobar en modelos celulares la posible sinergia de la administración concomitante de inhibidores de GSK3 y CK1, así como la combinación inhibidores de CK1 y TTBK1. Asimismo, se evaluará el efecto terapéutico de los fármacos duales preparados para este estudio. Para ello, se han realizado ensayos de viabilidad celular a diferentes concentraciones de los inhibidores, estudios del efecto neuroprotector del compuesto frente a la muerte neuronal inducida por el ácido etacrínico, y finalmente, cuantificación de la fosforilación de TDP-43 en cultivos de la línea celular SHSY-5Y. 


\section{Materiales y métodos}

\section{Inhibidores}

Los compuestos utilizados (stock $10 \mathrm{mM}$ ) son inhibidores de diferentes quinasas implicadas en la ELA, previamente sintetizados en el grupo de investigación y con actividad biológica probada. Los inhibidores duales fueron diseñados y sintetizados en el propio laboratorio del grupo de investigación como moléculas híbridas de dos inhibidores de proteínas quinasa diferente. De esta manera, el inhibidor dual $\mathrm{AB}$, es un compuesto híbrido con capacidad inhibitoria a nivel bajo micromolar en GSK-3 y CK1, que proviene de la unión de las moléculas A (inhibidor de GSK3) y B (inhibidor de CK1). También en este trabajo se ha evaluado la actividad biológica del compuesto multidiana $\mathrm{BC}$ con actividad inhibitoria en CK1 y TTBK1, diseñado a partir de las moléculas B y C (inhibidor de TTBK1) (Tabla 1).

\begin{tabular}{|c|c|c|c|c|}
\hline Estructura química & $\begin{array}{l}\text { Nombre } \\
\text { del } \\
\text { compuesto }\end{array}$ & $\begin{array}{l}\text { GSK- } \\
3 \text { CI } \\
(\mu \mathrm{M})\end{array}$ & $\begin{array}{l}\text { CK- } \\
1 \delta \\
\mathbf{C I}_{50} \\
(\mu \mathrm{M})\end{array}$ & $\begin{array}{l}\text { TTBK1 } \\
\text { CI I0 } \\
(\mu \mathrm{M})\end{array}$ \\
\hline & $\mathbf{A B}$ & 3.85 & 1.25 & -- \\
\hline & $\mathbf{A}$ & 2.01 & -- & -- \\
\hline & B & -- & 0.33 & -- \\
\hline & C & -- & -- & 0.52 \\
\hline & BC & -- & 17.48 & 0.11 \\
\hline
\end{tabular}

Tabla 1.- Estructuras químicas y valores de concentración inhibitoria 50 (CI50) sobre diferentes proteínas quinasas de los compuestos (sondas moleculares) utilizados en este trabajo.

\section{Cultivos de células neuronales}

Para la realización de los experimentos, se utilizó la línea celular de neuroblastoma humano denominada SH-SY5Y. Hay que destacar que estas células presentan propiedades adherentes que hay que tener en cuenta a la hora de realizar los ensayos. Las células se cultivan en medio DMEM (Dulbecco's Modified Eagle Medium) suplementado con 10\% de suero fetal bovino (FBS) y un $1 \%$ de penicilina/estreptomicina en incubador a $37^{\circ} \mathrm{C}$ y $5 \%$ de $\mathrm{CO}_{2}$. Cuando se consigue la confluencia óptima, las células se cultivan en placas de 96 pocillos. 


\section{Estudio de viabilidad celular de los inhibidores}

El ensayo de viabilidad celular se realiza para poder elegir la concentración de compuesto en los siguientes estudios de eficacia. Se evaluaron los inhibidores a diferentes concentraciones $(1 \mu \mathrm{M}, 5 \mu \mathrm{M}$, $10 \mu \mathrm{M})$ estudiándose la viabilidad celular con el ensayo de MTT (3- (4,5-dimetiltiazol-2-il) -2,5difeniltetrazolio). El MTT es un compuesto soluble y de color amarillo que pertenece a la familia de las sales de tetrazolio. El ensayo se fundamenta en la reducción mitocondrial del compuesto, lo que da lugar al formazán. Este compuesto perteneciente a la familia de los formazanos se acumula dentro de la célula como un precipitado insoluble y de color púrpura [14]. Para realizar su cuantificación se utiliza el disolvente orgánico DMSO (dimetilsulfóxido) y posteriormente se mide la absorbancia a $595 \mathrm{~nm}$.

Se cultivan 120.000 células/pocillo en una placa de 96 pocillos, a $37^{\circ} \mathrm{C}$ y $5 \% \mathrm{CO}_{2}$. Cuando se consigue la confluencia óptima de las células se procede a tratarlas a diferentes concentraciones de los inhibidores. Veinticuatro horas después se añade el MTT, y se incuba durante 4 horas, de esta manera, se consigue la precipitación del MTT y el viraje a morado. Pasado este tiempo, se procede a aspirar el medio y se incorpora el DMSO para producir la lisis celular así como la solubilización de los cristales. Finalmente, se mide la absorbancia a $595 \mathrm{~nm}$, utilizando para ello el lector de placas Multiskan Sky (Thermo Scientific $\left.{ }^{\mathrm{TM}}\right)$.

\section{Estudios de neuroprotección celular frente al ácido etacrínico}

Para ver el efecto neuroprotector de los compuestos se genera un modelo celular que simula uno de los procesos patológico desencadenantes de la enfermedad, en este caso, el aumento de la proteína TDP-43 fosforilada. Para realizarlo, se utiliza el ácido etacrínico (AE) que produce la depleción del glutatión. Esto se traduce en un aumento del estrés oxidativo, que favorece la fosforilación del carbono terminal de la serina en la posición 403/404 y 409/410 de la proteína TDP-43 [15].

La concentración de AE utilizado debe de ser aquella que produzca el 50\% de la muerte celular. Por lo que previamente a los estudios de neuroprotección se realiza ensayos de dosis-respuesta con ácido etacrínico. Se cultivan 120.000 células en placas de 96 pocillos a $37^{\circ} \mathrm{C}$ y $5 \%$ de $\mathrm{CO}_{2}$. Cuando se alcanza la confluencia celular óptima se añade $\mathrm{AE}$ (stock $70 \mathrm{mM})$ a diferentes concentraciones $(30 \mu \mathrm{M}, 40 \mu \mathrm{M}$, $50 \mu \mathrm{M}, 60 \mu \mathrm{M}$ y $70 \mu \mathrm{M}$ ) y se continúa realizando el ensayo de MTT descrito anteriormente.

Para la realización del experimento de neuroprotección se utilizan 120.000 células/pocillo en placas de 96 pocillos en condiciones de $37^{\circ} \mathrm{C}$ y $5 \%$ de $\mathrm{CO}_{2}$. Una vez que las células llegan a la confluencia óptima se tratan con las dosis correspondientes de los inhibidores. Además, se utiliza como control positivo del efecto neuroprotector, VP4.55. Este compuesto, es un inhibidor de GSK3 $\beta$ capaz de revertir el efecto producido por el AE y por lo tanto de producir neuroprotección celular. Pasada una hora del tratamiento, se añade el volumen necesario de $\mathrm{AE}$ (stock $70 \mathrm{mM}$ ) que produce el $50 \%$ de la muerte celular. En este caso, sería la incorporación de $10 \mu \mathrm{l}$ de EA $(50 \mu \mathrm{M})$. Veinticuatro horas después, se procede a realizar el ensayo de MTT.

\section{Análisis estadístico}

El análisis estadístico de los resultados se llevó a cabo mediante el programa GraphPad Prism (GraphPad Software, Inc.). El análisis cuantitativo se realizó con tres experimentos biológicamente independientes para la viabilidad celular y la neuroprotección, calculando la media \pm desviación estándar de la media y utilizando la T de Student de muestras independientes.

\section{Resultados y discusión}

\section{Viabilidad celular}

Para poder elegir las dosis de los estudios de neuroprotección, se hicieron previamente ensayos de viabilidad celular, con el propósito de descartar las concentraciones de los compuestos que producen una baja viabilidad celular y que por lo tanto desencadenan la muerte celular. Todos los inhibidores fueron ensayados a tres concentraciones diferentes $(1 \mu \mathrm{M}, 5 \mu \mathrm{M}, 10 \mu \mathrm{M})$. Los resultados obtenidos mostraron, que todos los compuestos de partida $(\mathrm{A}, \mathrm{B}, \mathrm{C})$ resultaron ser viables a las tres concentraciones evaluadas.

Sin embargo, en los compuestos multidiana, los resultados fueron diferentes. El derivado AB mostró una disminución en la viabilidad celular a las tres concentraciones del estudio. Mientras que el compuesto BC alcanzó únicamente niveles de viabilidad celular similares al control para la concentración de $1 \mu \mathrm{M}$. Hay que tener en cuenta, que al observar las células tratadas con los compuestos duales (multidiana), se aprecia la formación de agregados cristalinos debido a la precipitación del compuesto (Figura3). La cantidad de estos agregados aumenta conforme se incrementa la concentración de dicho compuesto. Este hecho podría ser el desencadenante de la muerte celular. Los compuestos multidiana tiene un peso molecular más elevado que los inhibidores individuales lo que varía diferentes propiedades físico químicas responsables de la disminución de la solubilidad. 

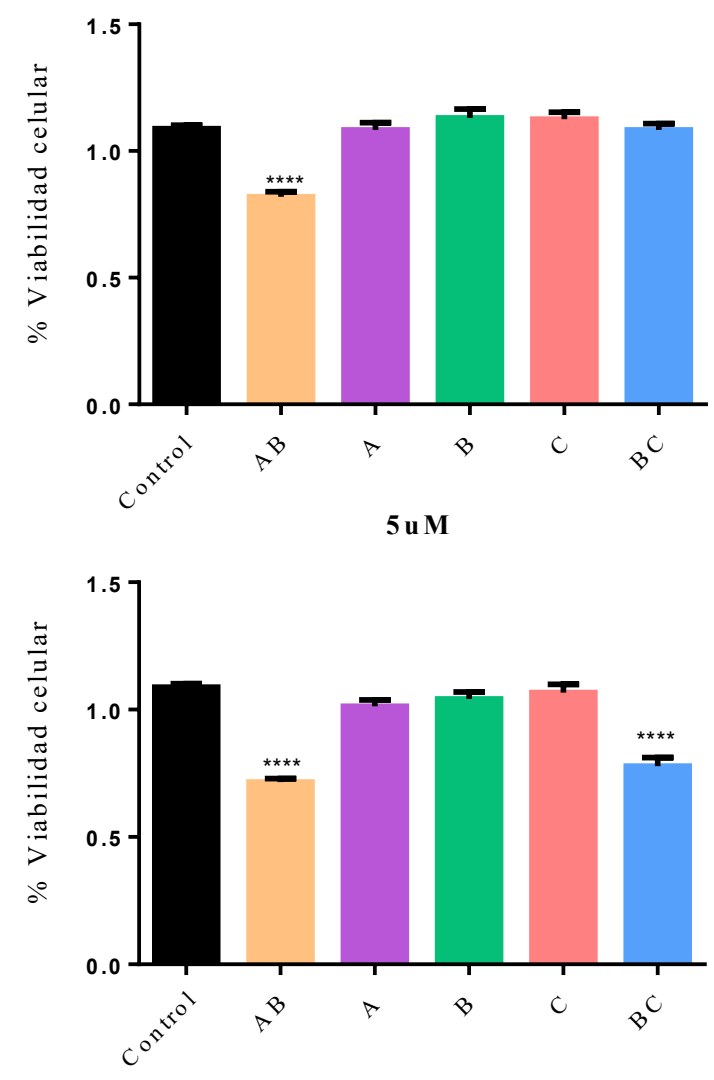

10 u M

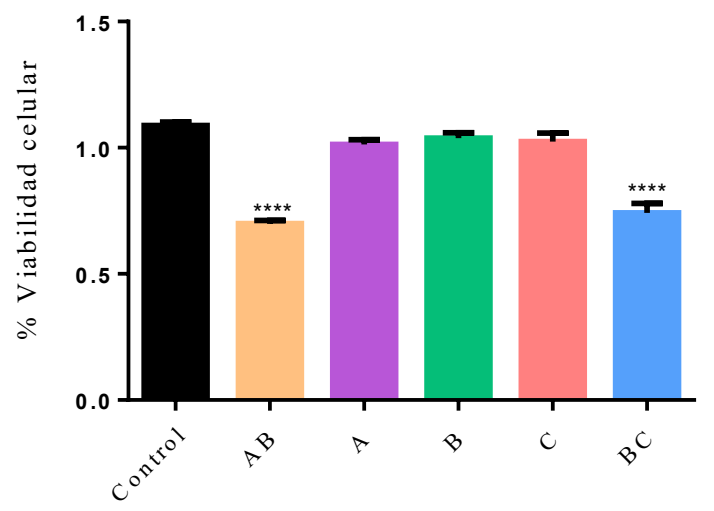

Figura 2.- Experimento de viabilidad celular. Estudio de toxicidad en la línea celular SHSY-5Y tras el tratamiento con los inhibidores $(1 \mu \mathrm{M}, 5 \mu \mathrm{M}, 10 \mu \mathrm{M})$ con respecto al control. Los datos que se muestran son la media \pm SEM de tres experimentos independientes. ( $* * * * p<0,0001$ diferencias significativas con respecto al control).

\section{Neuroprotección}

El objetivo del ensayo consiste en identificar la eficacia de los inhibidores de quinasas, sus mezclas binarias y los compuestos multidiana en la neuroprotección celular frente al efecto del ácido etacrínico. El experimento, se realizó solo con los compuestos que mantienen la viabilidad celular a la concentración de $1 \mu \mathrm{M}$. En este caso se trata del híbrido BC, que tiene una actividad dual inhibitoria en CK1 y TTBK1. También se utilizan los inhibidores de partida B y C, así como se evaluó el posible efecto sinérgico de la administración simultánea de los dos inhibidores a la vez $(B+C)$. De esta manera, se estudiaron los compuestos de partida $\mathrm{B}$ y $\mathrm{C}$ a $1 \mu \mathrm{M}$, el inhibidor dual $\mathrm{BC}$ a esta misma concentración $(1 \mu \mathrm{M})$, así como el tratamiento simultáneo con los compuestos $\mathrm{B}$ y $\mathrm{C}$ a concentraciones inferiores $(0.5 \mu \mathrm{M}+0.5 \mu \mathrm{M})$.

La figura 4 , recoge los resultados de este estudio, donde aunque el inhibidor dual $\mathrm{BC}$ y uno de sus compuestos iniciales $\mathrm{C}$, inhibidor de TTBK1, mostraron tendencia a la neuroprotección, únicamente $\mathrm{B}$ (inhibidor de CK1) y la suma del tratamiento simultaneo B + C, fueron capaces de proteger frente al EA de manera significativa. Por tanto, se confirma la sinergia de la inhibición simultánea de CK1 y TTBK1 
en la neuroprotección frente al AE, y por tanto el potencial efecto terapéutico que tiene la inhibición simultánea de varias dianas en enfermedades multifactoriales.

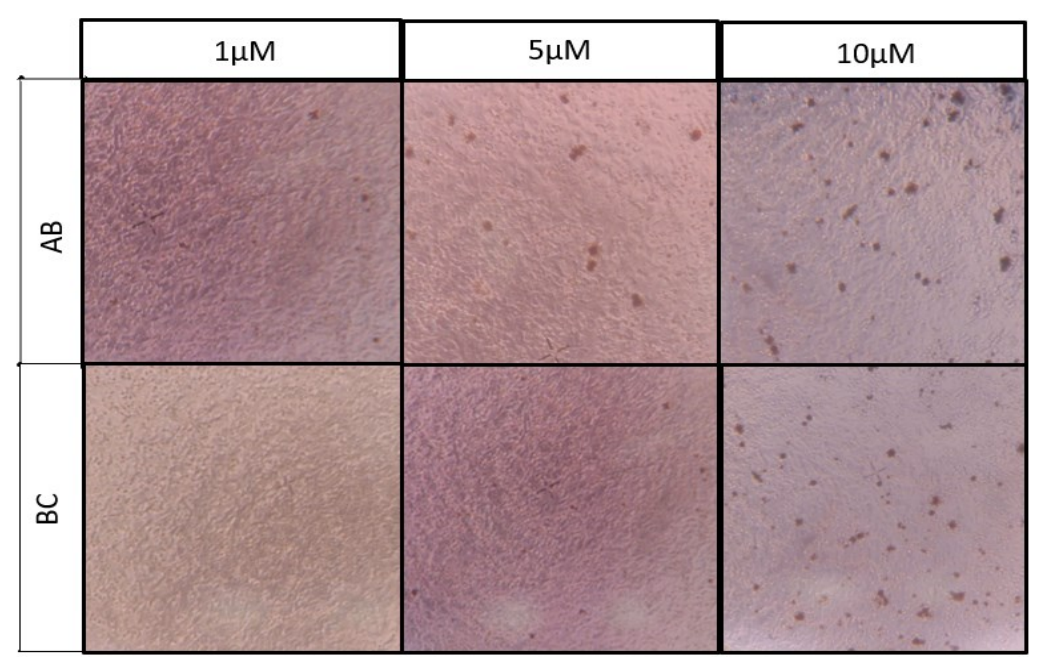

Figura 3.- Precipitados de los inhibidores duales $(\mathrm{AB}, \mathrm{BC})$ a diferentes concentraciones $(1 \mu \mathrm{M}, 5 \mu \mathrm{M}$, $10 \mu \mathrm{M})$.

\section{Neuroprotección}

El objetivo del ensayo consiste en identificar la eficacia de los inhibidores de quinasas, sus mezclas binarias y los compuestos multidiana en la neuroprotección celular frente al efecto del ácido etacrínico. El experimento, se realizó solo con los compuestos que mantienen la viabilidad celular a la concentración de $1 \mu \mathrm{M}$. En este caso se trata del híbrido BC, que tiene una actividad dual inhibitoria en CK1 y TTBK1. También se utilizan los inhibidores de partida $\mathrm{B}$ y $\mathrm{C}$, así como se evaluó el posible efecto sinérgico de la administración simultánea de los dos inhibidores a la vez $(B+C)$. De esta manera, se estudiaron los compuestos de partida B y C a $1 \mu \mathrm{M}$, el inhibidor dual $\mathrm{BC}$ a esta misma concentración $(1 \mu \mathrm{M})$, así como el tratamiento simultáneo con los compuestos $\mathrm{B}$ y $\mathrm{C}$ a concentraciones inferiores $(0.5 \mu \mathrm{M}+0.5 \mu \mathrm{M})$.

La figura 4, recoge los resultados de este estudio, donde aunque el inhibidor dual BC y uno de sus compuestos iniciales $\mathrm{C}$, inhibidor de TTBK1, mostraron tendencia a la neuroprotección, únicamente $\mathrm{B}$ (inhibidor de CK1) y la suma del tratamiento simultaneo B $+\mathrm{C}$, fueron capaces de proteger frente al EA de manera significativa. Por tanto, se confirma la sinergia de la inhibición simultánea de CK1 y TTBK1 en la neuroprotección frente al AE, y por tanto el potencial efecto terapéutico que tiene la inhibición simultánea de varias dianas en enfermedades multifactoriales.

$1 \mathbf{u M}+\mathbf{A} \mathbf{E} 50 \mathbf{M}$

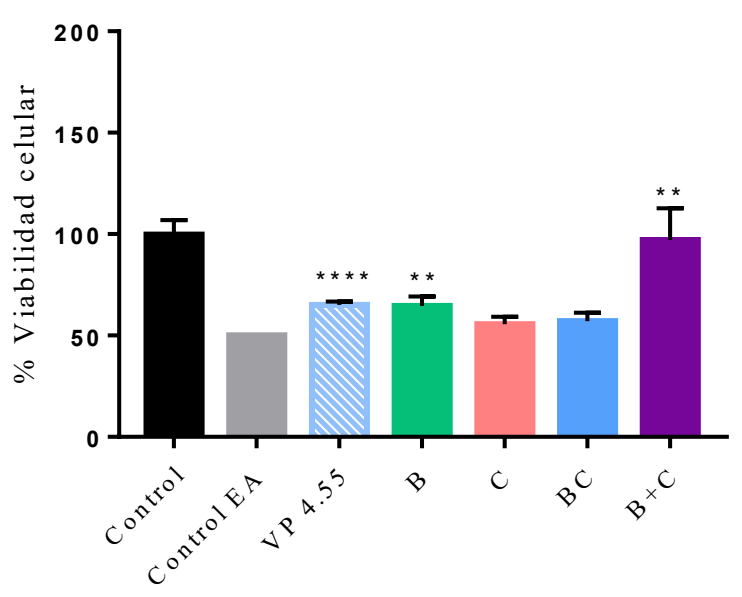

Figura 4.- Experimento de neuroprotección. Estudio de la capacidad de los compuestos $(1 \mu \mathrm{M})$ para revertir el efecto del ácido etacrínico $(50 \mu \mathrm{M})$ en la línea celular SHSY-5Y.Los datos que se muestran son la media \pm SEM de tres experimentos independientes. $\left({ }^{* *} \mathrm{p}<0,01 ; * * * \mathrm{p}<0,00001\right.$ diferencias significativas con respecto al control). 
Posteriormente, se decidió ensayar los mismos compuestos a una concentración más baja $(0.5 \mu \mathrm{M})$ y una administración simultanea de los dos inhibidores de CK1 y TTBK1 $(0.25 \mu \mathrm{M}+0.25 \mu \mathrm{M})$.

Como se puede observar en la Figura 5, el derivado multidiana BC a $0.5 \mu \mathrm{M}$ muestra una actividad neuroprotectora, contrarrestando el efecto producido por el EA de manera significativa. Este resultado es relevante, puesto que confirma la hipótesis de partida, en la que un tratamiento multifactorial es mejor que la monoterapia. Por otra parte, el efecto neuroprotector de $\mathrm{BC}$ es similar al obtenido por el tratamiento con la combinación $\mathrm{B}+\mathrm{C}$, lo que puede suponer una ventaja farmacológica en un posible tratamiento terapéutico al eliminar posibles interacciones medicamentosas y tener un perfil ADME más favorable.

Adicionalmente, también se estudió a concentración $0.5 \mu \mathrm{M}$ el compuesto multidiana $\mathrm{AB}$, un híbrido entre inhibidores de GSK-3 y de CK1. En este caso, dada la baja solubilidad del mismo en el medio de ensayo, no se logró observar un efecto neuroprotector, aunque si fue posible determinar el efecto sinérgico que la inhibición simultanea de GSK-3 y CK1 tienen frente al daño celular producido por el AE. En un futuro, se llevarán a cabo modificaciones estructurales en el compuesto híbrido para que manteniendo la doble actividad biológica, aumente la solubilidad en los medios biológicos y mejore sus propiedades farmacológicas.
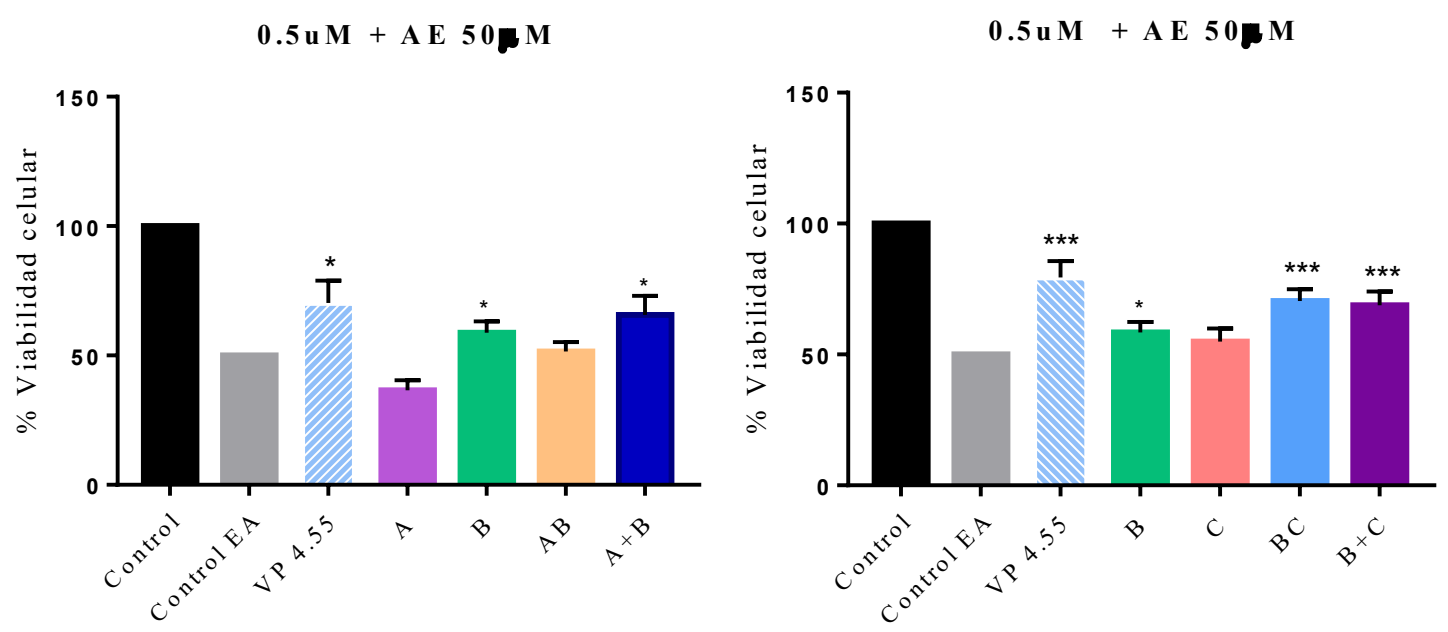

Figura 5.- Experimento de neuroprotección. Estudio de la capacidad de los compuestos $(0.5 \mu \mathrm{M})$ para revertir el efecto del ácido etacrínico $(50 \mu \mathrm{M})$ en la línea celular SHSY-5Y.Los datos que se muestran son la media \pm SEM de tres experimentos independientes. $(* \mathrm{p}<0.05, * * \mathrm{p}<0,01 ; * * * * \mathrm{p}<0,001$ diferencias significativas con respecto al control).

\section{Conclusiones}

Durante la realización de este trabajo, se ha estudiado el efecto terapéutico producido por la modulación simultánea de varias dianas implicadas en la patología de la esclerosis lateral amiotrófica. Los resultados experimentales han mostrado, que la combinación de inhibidores de GSK3 y CK1, o bien inhibidores de CK1 y TTBK1 presentan un potente efecto sinérgico en la neuroprotección celular frente al daño causado por el ácido etacrínico.

Además, hemos podido comprobar la eficacia de los fármacos multidiana en patologías complejas como la ELA, y así el compuesto dual BC, inhibidor de CK1 y TTBK1, es capaz de producir un efecto neuroprotector frente al efecto del ácido etacrínico. Por lo tanto, los fármacos multidiana pueden constituir una potencial estrategia terapéutica para las proteinopatías de TDP-43. Así mismo, hay que reflejar la importancia de comprobar la disminución de la fosforilación de TDP-43 por western-blot así como mejorar las propiedades fisicoquímicas de los compuestos para lograr una mejora en el perfil de solubilidad.

Posteriormente, habría que realizar estudios que demostraran la eficacia de este fármaco en células de pacientes con ELA y en modelos ratones que simulen la patología de esta enfermedad neurodegenerativa.

\section{Bibliografía}

1. Bayer, T. A. 2015. Proteinopathies, a core concept for understanding and ultimately treating degenerative disorders? European Neuropsychopharmacology. 25(5):713-24. 
2. Hardiman O, Al-Chalabi A, Chio A, Corr EM, Logroscino G, Robberecht W, Shaw PJ, Simmons Z, Van Den Berg LH. 2017. Amyotrophic lateral sclerosis. Nature Reviews Disease Primers. 5(3):17071.

3. Zarei S, Carr K, Reiley L, Diaz K, Guerra O, Altamirano PF, Pagani W, Lodin D, Orozco G, Chinea A. 2015. A comprehensive review of amyotrophic lateral sclerosis. Surgical neurology international. 6(1):171.

4. Lee E. B, Lee V. M, Trojanowski J. Q. 2012. Gains or losses: molecular mechanisms of TDP43mediated neurodegeneration. Nature Reviews Neuroscience. 13(1):38.

5. Prasad A, Bharathi V, Sivalingam V, Girdhar A, Patel B. K. 2019. Molecular Mechanisms of TDP-43 Misfolding and Pathology in Amyotrophic Lateral Sclerosis. Frontiers in molecular neuroscience. $12: 25$.

6. Saraswati, A. P, Hussaini, S. A, Krishna, N. H, Babu, B. N, Kamal, A. 2018. Glycogen synthase kinase-3 and its inhibitors: potential target for various therapeutic conditions. European journal of medicinal chemistry. 144:843-58.

7. Kametani, F, Nonaka, T, Suzuki, T, Arai, T, Dohmae, N, Akiyama, H, Hasegawa, M. 2009. Identification of casein kinase-1 phosphorylation sites on TDP-43. Biochemical and biophysical research communications. 382(2):405-9.

8. Alquezar C, Salado I.G, De la Encarnación A, Pérez D.I, Moreno F, Gil C, de Munain A.L, Martínez A, Martín-Requero Á. 2016. Targeting TDP-43 phosphorylation by Casein Kinase- $1 \delta$ inhibitors: a novel strategy for the treatment of frontotemporal dementia. Molecular neurodegeneration. 11(1):36.

9. Perez D.I, Gil C, Martinez A. 2011. Protein kinases CK1 and CK2 as new targets for neurodegenerative diseases. Medicinal research reviews. 31(6):924-54.

10. Taylor L.M, McMillan P.J, Kraemer B.C, Liachko NF. 2019. Tau tubulin kinases in proteinopathy. The FEBS journal. Disponible en: https://febs.onlinelibrary.wiley.com/doi/full/10.1111/febs.14866. Fecha consulta: 30/5/2019.

11. Bansal Y, Silakari O. 2014. Multifunctional compounds: smart molecules for multifactorial diseases. Eur J Med Chem. 76 (9):31-42.

12. Müller-Schiffmann A, Sticht H, Korth C. 2012. Hybrid compounds: from simple combinations to nanomachines. BioDrugs. 26(1):21-31

13. Bottegoni G, Favia AD, Recanatini M, Cavalli A. 2012. The role of fragment-based and computational methods in polypharmacology. Drug Discovery Today. 17(1-2):23-34.

14. Van Meerloo J, Kaspers GJ, Cloos J. 2011. Cell sensitivity assays: the MTT assay. Methods Mol Biol. 731:237-45.

15. Iguchi Y, Katsuno M, Takagi S, Ishigaki S, Niwa J.I, Hasegawa M, Tanaka F, Sobue G. 2012. Oxidative stress induced by glutathione depletion reproduces pathological modifications of TDP-43 linked to TDP-43 proteinopathies. Neurobiology of disease.45(3):862-70. 